\title{
Ecologia de comunidades das larvas de Trichoptera Kirby (Insecta) em dois córregos de primeira ordem da Serra dos Pireneus, Pirenópolis, Goiás, Brasil
}

\author{
Leandro Gonçalves Oliveira ${ }^{1}$ \\ Pitágoras da Conceição Bispo ${ }^{2}$
}

\begin{abstract}
Community ecology of Trichoptera Kirby (Insecta) larvae in two first-order streams of Serra dos Pireneus, Pirenópolis, Goiás, Brazil. The Trichoptera fauna in two first-order streams of Serra dos Pireneus, Goiás State, was studied from June/1993 to July/1994. The Trichoptera larvae abundances were lower in the rainy season in the two streams. The temporal distributions of the functional groups (feeding categories) was similar in both streams. The ordination by Detrended Correspondence Analysis (DCA) detected a higher temporal heterogeneity of the Trichoptera community in one of the sampling stations, probably associated with higher discharge variation. There is a large variation of abundance of some genera between the two studied streams.

KEY WORDS. Trichoptera, community, stream, feeding categories, detrended correspondence analysis
\end{abstract}

Os tricópteros são importantes organismos dentro do sistema lótico, sendo relevantes elos na transferência de energia dentro desses ecossistemas. Dentro da ordem estão incluídos membros de todas as categorias funcionais alimentares propostas por CUMmins \& KLUG (1979), sendo portanto, a ordem de insetos aquáticos mais diversificada do ponto de vista funcional (WiGgINS \& MACKAY 1978). No Brasil, alguns estudos sobre ecologia do grupo foram realizados por Oliveira \& FroeHLich (1996, 1997), Oliveira et al. (1999) e HuAMANTINCO \& NESSIMIAN (2000).

Os estudos ecológicos de comunidades de insetos aquáticos, normalmente, comparam comunidades submetidas a grupos de fatores ambientais muito diferentes, o que dificulta a individualização de quais desses fatores poderiam estar atuando na estruturação da comunidade. Neste caso, o que se obtêm são grupos de fatores que potencialmente poderiam estar explicando as diferentes comunidades. Nesse sentido, o objetivo do presente trabalho foi avaliar a estrutura de duas comunidades de larvas de Trichoptera em dois córregos de primeira ordem, ambientalmente semelhantes, ao longo de 14 meses, tanto em termos de composição quanto de grupos alimentares funcionais.

1) Departamento de Biologia Geral, Instituto de Ciências Biológicas, Universidade Federal de Goiás. Caixa Postal 131, 74001-970 Goiânia, Goiás, Brasil. Bolsista do CNPq.

2) Museu de Zoologia, Universidade de São Paulo. Caixa Postal 42694, 4299-970 São Paulo, São Paulo, Brasil. 


\section{MATERIAL E MÉTODOS}

\section{Área de estudo}

O trabalho foi realizado na bacia do Rio das Almas, Pirenópolis, Goiás, cujas coordenadas geográficas são: $15^{\circ} 51^{\prime} \mathrm{S}$ e $48^{\circ} 57^{\prime} \mathrm{W}$, conforme carta topográfica na escala de 1:100.000, do Ministério do Exército, folha SD 22-Z-D-V. O Rio das Almas nasce na Serra dos Pireneus e suas nascentes compreendem vários córregos encachoeirados, íngremes e com fundos de pedra, areia e algumas piscinas com folhiço. Estes tributários fluem na vertente ocidental da serra e fazem parte da rede hidrográfica da Bacia Amazônica.

Segundo NIMER (1989), a região estudada apresenta um clima tropical semi-úmido, com estação chuvosa no verão (grande pluviosidade entre dezembro e fevereiro) e uma estação seca no inverno (de maio a setembro).

$\mathrm{O}$ estudo foi conduzido em dois córregos de primeira ordem, segundo a classificação de STRAHLER (1957). O Córrego do Inferno (Ponto 1), localiza-se no alto da Serra dos Pireneus (1100 m) e o Córrego Vagafogo (Ponto 2), um afluente do Rio das Almas à jusante da cidade $(730 \mathrm{~m})$. Ambos os córregos possuem densa cobertura vegetal e nenhuma ação antrópica. Além disso, apresentam substratos pedregosos, com seixos de tamanhos variados, areia e silte, bem como uma grande quantidade de folhiço.

\section{Coletas e identificação taxonômica e das categorias funcionais}

As coletas foram realizadas mensalmente nos dois córregos supracitados, num período de 14 meses (junho/1993 a julho/1994). As larvas foram coletadas em regiões de corredeira com fundo pedregoso, com auxílio do amostrador de Surber ( $0,250 \mathrm{~mm}$ de malha). Uma vez por mês e em cada ponto de coleta, foram feitas 20 subamostras aleatórias, perfazendo um total de $2 \mathrm{~m}^{2}$ em cada amostragem. Após coletado, o material foi etiquetado e acondicionado em álcool a $80 \%$. No laboratório, esse material foi triado e as larvas de Trichoptera foram separadas e identificadas a nível de gênero, utilizando-se os trabalhos de WigGins (1977) e ANGRISANO (1995).

O enquadramento dos gêneros nas categorias alimentares funcionais propostas por Cummins \& KLUG (1979), foi feito com base na análise do conteúdo estomacal e de observações das peças bucais. Na análise do conteúdo estomacal, os tubos digestivos de no máximo cinco espécimes por gênero foram examinados para uma simples avaliação dos itens alimentares. No caso de Hydropsychidae Curtis, 1835, além de itens como algas, detritos e fragmentos de folhas e madeira, foram encontrados fragmentos de Arthropoda. Diante desse fato, as larvas dessa família foram consideradas coletoras (filtradoras), já que filtram partículas como detritos e até mesmo diatomáceas e outros animais (MERRITT \& CUMMINS 1996), coletando o que ficou preso em suas redes.

\section{Fatores Ambientais}

Fatores abióticos tais como precipitação pluviométrica, temperatura do ar e da água, velocidade, vazão, condutividade elétrica e potencial hidrogeniônico $(\mathrm{pH})$ da água, foram obtidos da mesma maneira em ambos os pontos (1. Córrego do Inferno e 2. Vagafogo). 
As temperaturas da água e do ar foram tomadas com auxílio de um termômetro a álcool $\left(0-50^{\circ} \mathrm{C}\right)$. A velocidade da água foi obtida através do método do flutuador e a vazão foi calculada através do produto da velocidade média da água pela secção feita no córrego, multiplicando-se a largura, em pontos fixos, pela média das profundidades (LIND 1979). A condutividade elétrica, o potencial de oxi-redução e o potencial hidrogeniônico $(\mathrm{pH})$ da água, foram obtidos através de um condutivímetro de campo Corning PS-17 e um pHmetro de campo Corning PS-15, respectivamente. Como não foi possível a obtenção dos valores de precipitação nos pontos 1 e 2, utilizou-se as médias mensais de precipitação da cidade de Pirenópolis, as quais foram obtidas junto ao $10^{\circ}$ Distrito Meteorológico do Ministério da Agricultura, Goiânia, Goiás.

\section{Análise de dados}

A matriz de abundância, utilizando dados dos dois pontos de coleta, foi logaritimizada $(\log x+1)$ para diminuir a influência dos táxons dominantes sobre a análise. Essa matriz foi submetida a Análise de Correspondência Distendenciada (DCA: Detrended Correspondence Analysis), sendo ordenadas simultaneamente as observações (meses) e as variáveis (táxons) (GAUCH 1995). Essa análise foi realizada através do programa PC-ORD (MCCUNE \& MEFFORD 1995).

\section{RESULTADOS E DISCUSSÃO}

Nos dois córregos estudados foram coletadas 5.523 larvas de Trichoptera, sendo 2.048 no ponto 1 e 3.475 no ponto 2 . Um total de 25 gêneros foram coletados, sendo Xiphocentron Brauer, 1870, coletado somente no ponto 1 e Triplectides Kolenati, 1859, apenas no ponto 2. Os táxons, suas abreviaturas, abundâncias e as categorias funcionais de cada gênero estão listados na tabela I.

Os dois córregos estudados apresentam fisionomias semelhantes, conforme os fatores ambientais que os caracterizam (Tab. II). Os fatores físico-químicos como temperatura, $\mathrm{pH}$ e condutividade, aparentemente não proporcionaram relevantes alterações na fauna de insetos aquáticos, dentro das variações registradas nos dois locais de coleta. Estes dois pontos se distinguiram um do outro basicamente pela diferenças de altitude e vazão (Tab. II; Fig. 1A).

Em ambientes tropicais, a sazonalidade ambiental é influenciada principalmente pelo regime anual de chuva (WOLDA 1988; FERREIRA \& FROEHLICH 1992; Oliveira \& Froehlich 1997; Huamantinco \& Nessimian 2000). No caso dos insetos aquáticos em ambientes lóticos, o aumento da vazão no período chuvoso pode provocar o aumento do carreamento dos organismos, diminuindo a abundância dos mesmos. Dentre os insetos aquáticos, Trichoptera é um dos grupos mais afetados por esse carreamento, devido à forma do corpo e pelo fato de alguns dos grupos mais freqüentes como Hydropsychidae, Helicopsychidae Ulmer, 1906, Glossosomatidae Wallengren, 1891 e Xiphocentronidae Ross, 1949, viverem totalmente expostos à correnteza (FAESSEL 1985; OlIVEIRA \& FroEHLICH 1997). Provavelmente, grupos como Ephemeroptera Haeckel, 1896 (FERREIRA \& FrOEHLICH 1992) e Plecoptera Burmeister, 1838 podem ter suas comunidades menos influenciadas pela estação chuvosa do que os Trichoptera, principalmente por apresentarem formas corporais deprimidas, o que os possibilita a exploração da camada estagnada da coluna d'água (STATZNER \& Holm 1982). 
Tabela I. Listagem de gêneros, abreviaturas e suas respectivas categorias funcionais de larvas de Trichoptera para os dois pontos de coleta na Serra dos Pireneus, Pirenópolis, Goiás. (CF) Categoria funcional alimentar, $(C)$ coletor, $(F)$ fragmentador, $(P)$ predador, $(R)$ raspador.

\begin{tabular}{|c|c|c|c|c|c|c|}
\hline \multirow{2}{*}{ Familias } & \multirow{2}{*}{ Gêneros } & \multirow{2}{*}{ Abreviaturas } & \multicolumn{4}{|c|}{ Pontos de coleta } \\
\hline & & & 1 & 2 & Total & $\mathrm{CF}$ \\
\hline Calamoceratidae & Phylloicus & Phy & 81 & 12 & 93 & $F$ \\
\hline Glossosomatidae & Protoptila & Prot & 55 & 40 & 95 & $\mathrm{R}$ \\
\hline Hydrobiosidae & Atopsyche & Atop & 57 & 51 & 108 & $P$ \\
\hline Helicopsychidae & Helicopsyche & Helic & 47 & 63 & 110 & R \\
\hline \multirow[t]{3}{*}{ Hydropsychidae } & Leptonema & Lept & 436 & 893 & 1329 & C \\
\hline & Macronema & Mac & 217 & 54 & 271 & C \\
\hline & Smicridea & Smic & 515 & 796 & 1311 & C \\
\hline \multirow[t]{6}{*}{ Hydroptilidae } & Hydroptila & Hydr & 12 & 94 & 106 & $R, F$ \\
\hline & Neotrichia & Neot & 214 & 126 & 340 & $\mathrm{R}, \mathrm{F}$ \\
\hline & Oxyethira & Oxye & 1 & 1 & 2 & $\mathrm{R}, \mathrm{F}$ \\
\hline & Alisotrichia & Alisot & 1 & 4 & 5 & $\mathrm{R}$ \\
\hline & Dicaminus & Dic & 11 & 92 & 103 & $\mathrm{R}, \mathrm{C}$ \\
\hline & Genero1 & Hidr1 & 2 & 2 & 4 & R \\
\hline \multirow[t]{6}{*}{ Leptoceridae } & Atanatolica & Atan & 9 & 10 & 19 & $\mathrm{R}, \mathrm{C}$ \\
\hline & Nectopsyche & Nect & 3 & 4 & 7 & R, C \\
\hline & Oecetis & $\mathrm{Oec}$ & 28 & 107 & 135 & $\mathrm{R}, \mathrm{C}$ \\
\hline & Setodes & Set & 1 & 1 & 2 & $P, C$ \\
\hline & Triplectides & Trip & 7 & 0 & 7 & $\mathrm{~F}$ \\
\hline & Gênero2 & Lept2 & 85 & 57 & 142 & $\mathrm{~A}, \mathrm{C}$ \\
\hline \multirow[t]{2}{*}{ Odontoceridae } & Barypenthus & Bar & 19 & 6 & 25 & $\mathrm{~F}$ \\
\hline & Marilia & Mar & 189 & 318 & 507 & $F$ \\
\hline Philopotamidae & Chimarra & Chim & 53 & 413 & 466 & C \\
\hline \multirow[t]{2}{*}{ Polycentropodidae } & Aff. Cyrnellus & Aff. $C$ & 1 & 3 & 4 & C \\
\hline & Polyplectropus & Poly & 4 & 324 & 328 & C \\
\hline Xiphocentronidae & Xiphocentron & Xiph & 0 & 4 & 4 & C \\
\hline Total & & & 2048 & 3475 & & \\
\hline
\end{tabular}

Tabela II. Caracterização dos pontos de coleta das larvas de Trichoptera na Serra dos Pireneus, Pirenópolis, Goiás. A temperatura da água e do ar, velocidade, vazão e pH são média e desvio padrão dos registros dos 14 meses de coleta. Os valores de condutividade elétrica são dados em intervalos nos quais os valores ocorreram.

\begin{tabular}{lcc}
\hline \multirow{2}{*}{ Parâmetros } & Ponto 1 & Ponto 2 \\
\cline { 2 - 3 } & Córrego do Inferno & Córrego Vagafogo \\
\hline Ordem & Primeira & Primeira \\
Cobertura vegetal & Intensa & Intensa \\
Altitude $(\mathrm{m})$ & 1100 & 710 \\
Temperatura da Água $\left({ }^{\circ} \mathrm{C}\right)$ & $18,60 \pm 1,21$ & $20,64 \pm 1,99$ \\
Temperatura do $\mathrm{Ar}\left({ }^{\circ} \mathrm{C}\right)$ & $20,60 \pm 1,92$ & $22,07 \pm 2,63$ \\
Velocidade da Água $(\mathrm{m} / \mathrm{s})$ & $0,29 \pm 0,08$ & $0,37 \pm 0,13$ \\
Vazão $\left(\mathrm{m}^{3} / \mathrm{s}\right)$ & $0,04 \pm 0,02$ & $0,26 \pm 0,17$ \\
pH & $7,40 \pm 0,26$ & $7,54 \pm 0,21$ \\
Condutividade Elétrica $(\mu \mathrm{S} / \mathrm{cm})$ & $0-19$ & $10-29$ \\
\hline
\end{tabular}

Os dois pontos estudados são córregos pequenos (primeira ordem) e ambientalmente mais "estáveis" que outros córregos maiores nessa mesma região (terceira e quarta ordens) (L.G. Oliveira dados não publicados), sendo o ponto 1, córrego com menor vazão (Fig. 1A), o mais estável dos dois córregos estudados. Apesar da estabilidade, baixas abundâncias tenderam a ser registradas na estação chuvosa (dezembro a março) demonstrando que, pelo menos em parte, a variação anual da precipitação pluviométrica pode explicar a variação sazonal das larvas. Por outro

Revta bras. Zool. 18 (4): 1245 - 1252, 2001 


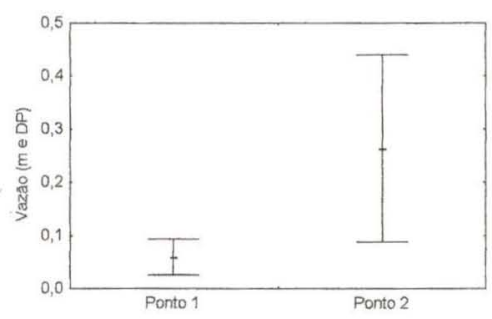

A: Variação da Vazão

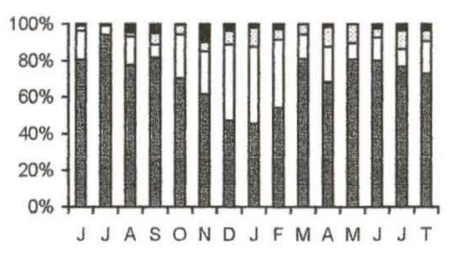

国 Coletor $\square$ Fragmentador $\square$ Raspador $\mathbf{E r e d a d o r}$

C: Ponto 1 , categorias funcionais

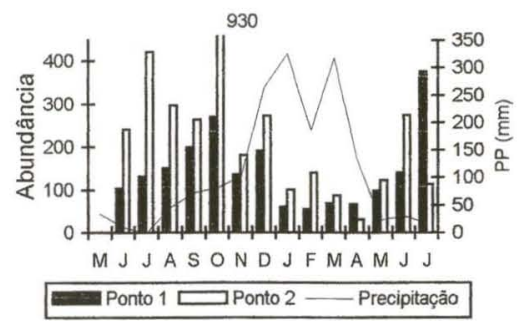

B: Variação da precipitação e da abundância

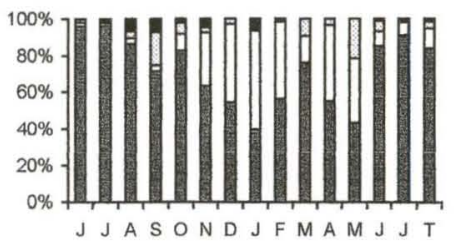

圆 Coletor $\square$ Fragmentador $\mathbf{\square}$ Raspador $\mathbf{E}$ Predador

D: Ponto 2, categorias funcionais

Fig. 1. Parâmetros analisados para larvas de Trichoptera coletadas nos pontos 1 e 2, no período de junho/1993 a julho/1994, na região de Pirenópolis, Goiás. (A) Média (m) e desvio padrão (DP) da variação anual da vazão nos pontos de coleta; (B) valores da média mensal da precipitação pluviométrica (PP) de maio/1993 a julho/1994 e variação temporal; (C-D) partição mensal dos grupos funcionais. (T) Total.

lado, foram observadas também altas flutuações de abundância na estação de seca. Provavelmente, nessa estação, quando a estabilidade ambiental é maior, essas flutuações na abundância poderiam ser atribuídas a outros fatores como disponibilidade de recursos, competição, predação e ou ciclo de vida. É importante salientar que a menor amplitude de variação da abundância no ponto 1 (55-376 indivíduos) em relação ao ponto 2 (31-930 indivíduos), também pode estar associada à maior estabilidade do primeiro ponto.

A distribuição das categorias funcionais para cada ponto indica que os raspadores e predadores foram grupos pouco freqüentes nos dois locais de coleta (Tab. I). Esse fato era esperado, uma vez que os pontos são intensamente sombreados, não permitindo a entrada de luz, consequentemente, prejudicando o desenvolvimento de perifiton utilizado como alimento pelos raspadores. Além disso, os predadores, na maioria das vezes ocorrem em baixo número (VANNOTE et al. 1980).

A categoria funcional mais freqüente foi a dos coletores, por volta de $80 \%$ dos organismos coletados em cada ponto (Tab. I). Este grupo é sempre freqüente em todos os córregos e tende a ser a única categoria funcional em córregos de altas ordens (VANNOTE et al. 1980). Os fragmentadores representaram 18 e $11 \%$ dos organismos coletados nos pontos 1 e 2, respectivamente. Esses organismos são beneficiados pela grande entrada de material alóctone da vegetação marginal (Cummins et al. 1989). Coletas realizadas nesses mesmos pontos, por unidade de 

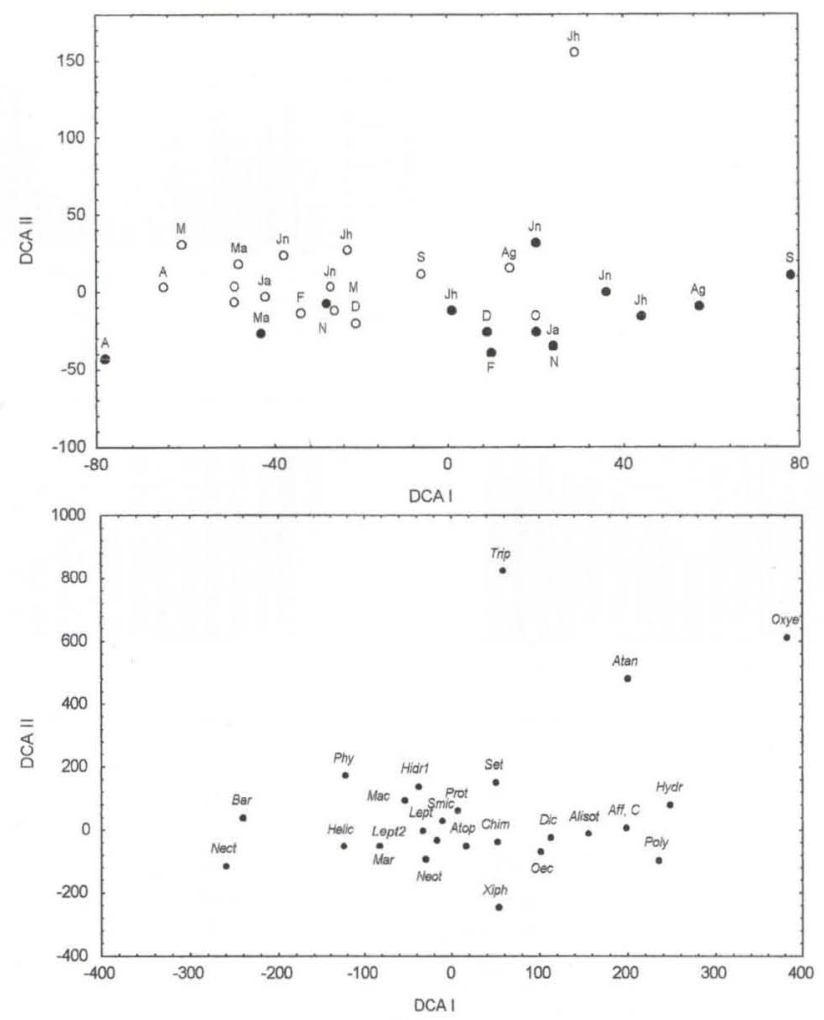

Fig. 2. Ordenação das observações, baseada na fauna de larvas de Trichoptera colecionada de junho/1993 a julho/1994, em dois pontos de coleta. (círculo vazio) Ponto 1, (círculo cheio) ponto 2. Ordenação dos táxons de Trichoptera colecionados de junho/1993 a julho/1994, nos dois pontos de coleta. Baseado na DCA. (Jn) Junho, (Ag) agosto, (S) setembro, (O) outubro, (N) novembro, (D) dezembro, (Ja) janeiro, (F) fevereiro, (Ma) março, (A) abril, (M) maio.

esforço de uma hora, utilizando-se redes circulares, verificaram maiores freqüências relativas de fragmentadores (OLIVEIRA et al. 1999). Isso provavelmente, pode ser devido a diferença de metodologias, já que o amostrador de Surber foi utilizado no presente trabalho basicamente para amostrar pedras e o folhiço coletado apenas naqueles acúmulos sob ou atrás das mesmas.

Os gêneros pertencentes às famílias Hydroptilidade Stephens, 1836, e Leptoceridae Leach, 1815, corresponderam a aproximadamente $14 \%$ das larvas de Trichoptera coletadas, porém nos gráficos da figura $1 \mathrm{C} \mathrm{e} \mathrm{D}$, não foram consideradas devido a dificuldade de encaixá-las seguramente em uma única categoria funcional, exceto o fragmentador Triplectides (Leptoceridae).

As distribuições das categorias funcionais durante os meses mostram que houve uma tendência nos dois pontos, da frequiência relativa dos coletores diminuir e dos fragmentadores aumentar na estação chuvosa (Fig. 1C, D). Essa modificação da frequêencia proporcional das duas categorias funcionais demonstra que os coletores foram mais sensíveis à sazonalidade ambiental provocada pelo regime anual 
de chuva do que os fragmentadores, o que pode ser atribuído ao fato deles estarem associados a depósitos de folhiço, que somente se acumulam em microhabitats mais estáveis. As distribuições temporais dos grupos funcionais se mostraram similares para os dois pontos (Fig. 1C, D).

A DCA mostra que, temporalmente, há uma maior heterogeneidade de comunidades de larvas de Trichoptera no ponto 2, quando comparada com o ponto 1, principalmente ao longo do primeiro eixo (Fig. 2). A maior variação da vazão no ponto 2, provavelmente, submeteu a fauna a maiores distúrbios ambientais do que no ponto 1, onde a estabilidade ambiental foi maior. Organismos como Phylloicus Mueller, 1880, Macronema Pictet, 1836, Neotrichia Morton, 1905, Triplectides e Barypenthus Burmeister, 1839, ocorreram em maior número no ponto 1, enquanto que Hydroptila Dalman, 1819, Dicaminus Mueller, 1879, Oecetis McLachlan, 1877, Marilia Mueller, 1880, Chimarra Stephens, 1829, Polycentropus Curtis, 1835 e Xiphocentron, ocorreram preferencialmente no ponto 2 (Tab. I; Fig. 2). Os resultados demonstraram que apesar da similaridade ambiental, as abundâncias foram distintas entre os dois córregos estudados.

AGRADECIMENTOS. A Vera L. Crisci pelas discussões sobre ecologia de insetos aquáticos e pela leitura crítica do manuscrito. A FUNAPE-UFG pelo apoio financeiro durante os trabalhos de campo. Ao CNPq e à FAPESP, pelas bolsas concedidas aos autores, respectivamente.

\section{REFERÊNCIAS BIBLIOGRÁFICAS}

Angrisano, E.B. 1995. Insecta Trichoptera, p. 1199-1224. In: E.C. Lopretto \& G. Tell (Eds). Ecosistemas de Aguas Continentales. Metodologias para su Estudio. La Plata, Argentina, Ediciones Sur, Vol. 3, XVIII+504p.

Cummins, K.W. \& M.J. KLuG. 1979. Feeding ecology of stream invertebrates. Ann. Rev. Ecol. Syst. 10: $147-172$.

Cummins, K.W.; M.A. Wilzbach; D.M. Gates; J.B. Perry \& W.B. Taliafero. 1989. Shredders and riparian vegetation. Bioscience 39: 24-30.

Faessel, B. 1985. Les Trichoptéres. Donnés biologiques, éthologiques et écologiques. Clés de determination larvaire des principaux genres de France. Bull. Fr. Pêche Piscic 299: 1-41.

Ferreira, M.J.N. \& C.G. Froehlich. 1992. Estudo da Fauna Ephemeroptera (Insecta) do Córrego do Pedregulho (Pedregulho, SP, Brasil) com aspectos da biologia de Thraulodes schlingeri Traver \& Edmunds, 1967. Revta bras. Ent. 36 (3): 541-548.

Gauch JR., H.G. 1995. Multivariate Analysis in Community Ecology. Cambridge, Univ. Press, $\mathrm{VIII}+298 \mathrm{p}$.

Huamantinco, A. \& J.L. Nessimian. 2000. Variation and life strategies of the Trichoptera (Insecta) larvae community in a first order tributary of the Paquequer River, Southeastern Brazil. Rev. Brasil. Biol. 60 (1): 73-82.

Lind, O.T. 1979. Handbook of Common Methods in Limnology. London, C.V. Mosby Co., 199p.

MCCune, B \& M.J.MefFord. 1995. PC-ORD. Multivariate Analysis of Ecological Data. Version 2.0. Oregon, MjM Software.

Merritt, R.W. \& K.W. Cummins. 1996. An Introduction to the Aquatic Insects of North America. Iowa, Kendall/Hunt Publ. Co., $3^{\text {rd }}$ ed. XIII+862p.

NIMER, E. 1989. Climatologia do Brasil. Rio de Janeiro, IBGE, 421p.

Oliveira, L.G. \& C.G. FroeHLICH. 1996. Natural history of three Hydropsychidae (Trichoptera, Insecta) in a "cerrado" stream from northeastern São Paulo, Brazil. Revta bras. Zool. 13 (3): 755-762. 
1997. The Trichoptera (Insecta) fauna of a "cerrado" stream in southeastern Brazil. Naturalia 22: 183-127.

OliveirA, L.G.; P.C. Bispo; V.L. CRISCI \& K.G. SousA. 1999. Distribuições de categorias funcionais alimentares de larvas de Trichoptera em uma região serrana do Brasil Central. Acta Limnol. Bras. 11 (2): 173-183.

STATZNER, B. \& T.F. Holm. 1982. Morphological adaptations of benthic invertebrates to stream flow an old question studied by means of a new technique (Laer Doppler Anenometry). Oecologia 53: 290-292.

STRAHLER, H.N. 1957. Quantitative analysis of watershed geomorphology. Amer. Geophys. Unio Trans. 33: 913-920.

Vannote, R.L.; G.W. Minshall; K.W. Cummins; J.R. Sedell \& C.E. Cushing. 1980. The River Continuum Concept. Can. Jour. Fish. Aquat. Sci. 37: 130-137.

WigGins, G.B. 1977. Larvae of the North America Caddisfly Genera (Trichoptera). Toronto, Univ. Toronto Press, $\mathrm{XI}+401 \mathrm{p}$.

WigGins, G.B. \& R.J. MACKAY. 1978. Some relationships between systematics and trophic ecology in Neartic aquatic insects, with special reference to Trichoptera. Ecology 59 (6): 1211-1220.

WoldA, H. 1988. Insect seasonality: Why? Ann. Rev. Ecol. Syst. 19: 1-18.

Recebido em 08.III.2001; aceito em 26.X.2001. 